\title{
Rational Design of Magnetic Topological Insulators
}

1. Magnetic-field-induced quantized anomalous Hall effect in intrinsic magnetic topological insulator $\mathrm{MnBi}_{2} \mathrm{Te}_{4}$ Authors: Yujun Deng, Yijun Yu, Meng Zhu Shi, Jing Wang, Xian Hui Chen and Yuanbo Zhang

arXiv:1904.11468

2. Quantum phase transition from axion insulator to Chern insulator in $\mathrm{MnBi}_{2} \mathrm{Te}_{4}$ Authors: Chang Liu, Yongchao Wang, Hao Li, Yang Wu, Yaoxin Li, Jiaheng Li, Ke He, Yong Xu, Jinsong Zhang, Yayu Wang arXiv:1905.00715

3. Quantum anomalous Hall effect driven by magnetic proximity coupling in all-telluride based heterostructure Authors: Ryota Watanabe, Ryutaro Yoshimi, Minoru Kawamura, Masataka Mogi, Atsushi Tsukazaki, Xiuzhen Yu, Kiyomi Nakajima, Kei S Takahashi, Masashi Kawasaki, Yoshinori Tokura arXiv:1908.07163

\section{Recommended with a Commentary by Liang Fu, MIT}

Chern insulator that exhibits quantized Hall effect from topologically nontrivial bands rather than Landau levels, can be viewed from a theoretical perspective as the mother of all topological insulators. Its first experimental realization in 2013 [1] is a remarkable achievement that relies on doping a non-magnetic topological insulator with magnetic atoms to a precisely controlled level of concentration, a challenge in material growth. Now, the highlighted papers present alternative approaches to make Chern insulators by rational design.

These new Chern insulators are made from two ingredients: (1) the topological insulator $\mathrm{Bi}_{2} \mathrm{Te}_{3}$ and (2) the magnetic insulator MnTe or (Zn,Cr)Te. The topological insulator provides two-dimensional Dirac states on both top and bottom surfaces. In the pristine state, the spin of these Dirac electrons is locked to their momentum by spin-orbit coupling. The magnetic insulator produces an out-of-plane exchange field that couples to Dirac electrons, cants their spin and opens an energy gap at the Dirac point. When the Fermi level is tuned into the gap, the massive Dirac states on each surface have a Chern number $\pm 1 / 2$, whose sign is determined by the direction of the local magnetization. When the magnetizations on top 
and bottom surfaces align, the two Chern numbers add up to \pm 1 , giving rise to quantized Hall conductance $\pm e^{2} / h$.

While this idea is simple, it is anything but easy to find the combination of itinerant Dirac electrons and local magnetic moments within a single material, let alone to coax them into cooperation. In the first generation of Chern insulators [1], this was achieved by doping $\mathrm{Bi}_{2} \mathrm{Te}_{3}$ with a high level $(\sim 15 \%)$ of randomly distributed magnetic dopants $\mathrm{Cr}$, which limits the ferromagnetic Curie temperature and degrades electron mobility.

In one of the highlighted papers, Watanabe et al successfully designed a Chern insulator by sandwiching a topological insulator $(\mathrm{Bi}, \mathrm{Sb})_{2} \mathrm{Te}_{3}$ layer between two ferromagnetic insulator layers $(\mathrm{Zn}, \mathrm{Cr}) \mathrm{Te}$. In this heterostructure, the magnetic proximity coupling at the interface drives the anomalous Hall effect, which becomes quantized at low temperature. The powerful technique of heterostructure engineering opens exciting possibilities, such as designing chiral electrical circuits and making 3D Chern superlattices.

In the other two papers, Deng et al and Liu et al made Chern insulators by exfoliating an intrinsic magnetic insulator $\mathrm{MnBi}_{2} \mathrm{Te}_{4}$ to atomically thin layers. $\mathrm{MnBi}_{2} \mathrm{Te}_{4}$ is a layered van der Waals crystal that can be viewed as a stack of alternating $\mathrm{Bi}_{2} \mathrm{Te}_{3}$ layer and $\mathrm{MnTe}$ layer. Below 25K, the Mn spins are ferromagnetically ordered within each layer with an out-of-plane easy axis, but are anti-parallel on adjacent layers; therefore bulk $\mathrm{MnBi}_{2} \mathrm{Te}_{4}$ is an antiferromagnetic insulator $[2,3]$. The authors realized a ferromagnetic Chern insulator in exfoliated few-layer $\mathrm{MnBi}_{2} \mathrm{Te}_{4}$, when an external magnetic field aligns all the spins and electrostatic gating brings the Fermi level inside the exchange gap.

Despite the use of a magnetic field, the quantized Hall conductance in $\mathrm{MnBi}_{2} \mathrm{Te}_{4}$ does not change sign as the carrier changes from electron to hole type, ruling out the scenario of Landau level quantization. In support of its true origin in intrinsic magnetic order, Deng et al found a sizable anomalous Hall effect already at zero magnetic field in three- and five-layer $\mathrm{MnBi}_{2} \mathrm{Te}_{4}$, presumably because of an unbalanced net magnetization in an odd number of layers. There is reason to hope that quantization will occur at higher temperature and smaller field as the sample quality improves. More broadly, the recent finding of massive Dirac electrons in a growing family of ferromagnets holds great promise for magnetic topological insulators [4].

The two generations of Chern insulators, using magnetic dopants versus heterolayers (artificial or natural), are like pizza and burger. The same ingredients can give us different tastes. The newly designed Chern insulators with robust/intrinsic magnetic order is likely to open new directions for research and potential applications. I can think of a few. The strong coupling between topological electrons and magnetism may enable electrical generation and manipulation of spin waves and skrymions. Due to the sizable exchange gap (a few tens of meV), a large or quantized Hall effect may be found at terahertz and infrared frequency, which is useful for optical isolation. Coupling magnetic topological insulator to superconductor provides a promising route to Majorana fermions [5, 6].

As is often the case in science, rational design goes hand in hand with serendipitous discovery. Very recently, Chern insulators were discovered unexpectedly in multilayer graphene $[7,8,9]$, an all-carbon system without magnetic atom! This fascinating story deserves highlight on its own. Suffice it to say, what was once serendipitous will become the basis for rational design in the future. 


\section{References}

[1] C.-Z. Chang et al., Experimental Observation of the Quantum Anomalous Hall Effect in a Magnetic Topological Insulator, Science, 340, 167 (2013).

[2] M. M. Otrokov et al., Prediction and observation of the first antiferromagnetic topological insulator, arXiv:1809.07389

[3] Y. Gong et al., Experimental realization of an intrinsic magnetic topological insulator, arXiv:1809.07926

[4] L. Ye et al, Massive Dirac fermions in a ferromagnetic kagome metal, Nature 555, 638 (2018)

[5] A. R. Akhmerov, Johan Nilsson, C. W. J. Beenakker, Electrically detected interferometry of Majorana fermions in a topological insulator, Phys. Rev. Lett. 102, 216404 (2009)

[6] L. Fu and C. L. Kane, Probing Neutral Majorana Fermion Edge Modes with Charge Transport, Phys. Rev. Lett. 102, 216403 (2009)

[7] Aaron L. Sharpe et al, Emergent ferromagnetism near three-quarters filling in twisted bilayer graphene, Science 365, 605 (2019)

[8] M. Serlin et al, Intrinsic quantized anomalous Hall effect in a moiré heterostructure, arXiv:1907.00261

[9] Guorui Chen et al, Tunable Correlated Chern Insulator and Ferromagnetism in Trilayer Graphene/Boron Nitride Moiré Superlattice, arXiv:1905.06535 